\title{
Analytical Model of Olfactory Sensory Information in Robot Odor Source Localization
}

\author{
Zhou Jie-Yong ${ }^{1,2}$, Xie Jia-Jia ${ }^{1,2}$ \\ ${ }^{1}$ School of Automation and Electrical Engineering, Tianjin University of Technology and Education, Tianjin, China \\ ${ }^{2}$ Tianjin Key Laboratory of Information Sensing and Intelligent Control, Tianjin, China
}

\begin{abstract}
The main purpose of establishing the analytical model of olfactory sensory information is to utilize measuring gas concentration of the current moment and wind speed and direction information in recent time to in local area to give odor source's existence measurement (yes, no, or unknown). It is generally known that gas sensor is contact measuring device, which can only measure the gas concentration of its space position, and cannot perceive at a distance. Besides, the spread of the gas mainly relies on environment airflow transport. The environmental airflow motion model should be established firstly so that to quantifiably judge whether there is an odor source in the robot's local territory.
\end{abstract}

Keywords: Odor source localization, Mobile robot, the analytical model of olfactory sensory information.

\section{Introduction}

Since the 1990s, some researchers use mobile robot carrying gas sensors to realize the functions of searching and determining the odor source, the study is called robot odor source localization, also known as active olfaction or chemical plume tracking [1].

Now, the robot odor source localization methods can be classified into three kinds in general. The first kind is chemical plume tracking [2] or robot active olfactory research influenced and produced by activities such as feeding, mating, and information communication. The branch focuses on the research of search behavior for odor source, which includes searching, tracking, and determining the source for odor or gas clues in the environment. The study has received the widespread attention because of the profound bionics background, of which typical methods include chemotaxis [3], Anemotaxis [4-5], infotaxis algorithm [6], fluxotaxis algorithm [7], the method that uses the online estimation of smell package's path to guide the robot to track plume [8], etc. The so-called plume refers to the spatial distribution of like feathers formed by the molecules released from the odor source diffusing in the air or water [9].

The second kind uses mobile robot for gas concentration distribution and mapping, and then, determine the position of the odor source, of which typical methods include the odor source localization based on bayesian occupancy grid mapping method [10], the localization method adopting Simple physical ideas to construct flow field model [11], concentration field surveying and mapping method based on multiple robots, Surveying and mapping method based on gaussian markov random field theory [12], etc.

The third kind is the combination of the first two kinds of methods, of which typical methods include using bayesian inference [13], fuzzy logic and particle filter [14] to estimate the position of the odor source, or using DAPSO algorithm, BFO algorithm, ACO algorithm [15]to survey and map odor concentration field in the process of robots' searching odor source.
According to the above methods of robot odor source localization, robotic active smell is the study of how to use smell information for odor source localization effectively. This requires that we must understand the transmission mechanism of odor, so as to establish the best analytical model of olfactory sensory information.

\section{Analysis of Olfactory Sensory Information}

\subsection{The Air Quality Mass}

In this paper, the concept of "air quality mass" is used to describe the environment fluid (air), which assumes that the environment fluid consists of much air quality mass, and environmental fluid movement expressed by each movement of the air quality mass. The so-called "air quality mass" refers to the set of air molecules having close relative position and relatively stable air molecules in the process of air movement. Air quality mass can envelop the target gases molecules emitted from the odor source. The air quality mass, released by the odor source, spread with the movement of turbulence, so the path of air quality mass must contain some odor source's location information, and the information can guide robot to track and confirm the odor source well.

The research only pay attentions to air quality mass arrived in robot location at each sampling instant, and estimate the probability of that where the air quality masses come from (air quality mass movement of the inverse model). All the space positions, that air quality mass may pass over before reaching the robots, are defined as air quality mass path. Air quality mass path estimation refers to the turbulent diffusion theory. It is a kind of inverse time axis application of turbulent diffusion theory, which uses historical wind speed and direction information, refers to long gaussian probability model, short diffusion model, etc. It is important to note that the estimated air quality mass path is not a curve, but a area where air quality mass pass shown by probability value characterization.

Suppose that the gas leakage rate is constant. The gas concentration measured by robot and the frequency and amplitude of concentration values in time domain, have 


\section{International Journal of Science and Research (IJSR) \\ ISSN (Online): 2319-7064}

Index Copernicus Value (2013): 6.14 | Impact Factor (2015): 6.391

contained the odor source intensity and the distance information between odor source and the robot. If the air quality mass contacting with the gas sensor carries enough target gas molecule, that is characterized by high concentration, so the each position of the scope covered by air quality mass path has the possibility of odor source existence (because of the unknown time of the target gas molecule group released from the odor source). If there's not enough gas molecules to be carried (below the detection limit of gas sensor), that is characterized by the undetected target gas, so it can be inferred that there may not be odor source in the scope covered by air quality mass path. So there is reason to infer that the air quality and the group path may not exist.

\subsection{The analytical model of sensing information}

Air quality mass motion model is established on the basis of the hypothesis that the distribution of the plume on the average wind direction in a long time obeys normal distribution. The specific expression is that, in the outdoor environment, the flow direction of atmosphere flow field changes in real time, the flow direction and velocity in a certain area is consistent at a particular time period. In the reference [14], Li Jigong has verified the hypothesis and has observed that when wind speed is not less than $0.2 \mathrm{~m} / \mathrm{s}$, the distance is not more than $10 \mathrm{~s}$ of displacement with the speed of the wind, flow field meet approximate uniform hypothesis. So the plume probability distribution within 10s obeys the law of the following formula.

$$
p(r)=\frac{1}{\sqrt{4 \pi D y}} \exp \left[-\frac{x^{2}}{4 D y}\right]
$$

Where, $r$ is the location of the robot's point, its value is $r=(x, y) ; D$ Said is vortex diffusion rate.

The plume probability distribution within 10 s is the farther distance from odor source in average wind direction, the smaller the probability; on the direction perpendicular to the average wind direction, the smoke plume probability obeys normal distribution, its specific probability distributions of the plume as shown in Figure 1.

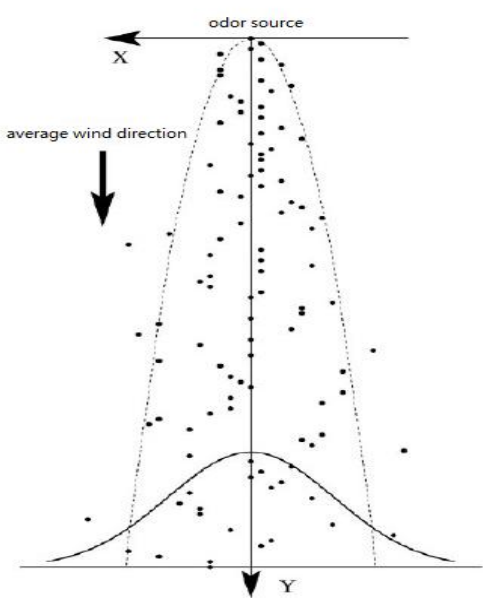

Figure 1: The probability distributions of the plume

The analytical model of olfactory sensory information is to estimate the inverse model of air quality mass's path that refers to the turbulent diffusion theory. It is a kind of inverse time axis application of turbulent diffusion theory, which uses odor concentration and historical wind speed and direction information, refers to Balkovsky plume model. Robots have measured odor information at some point in the search area, it can be concluded that the measured odor source is in the wedge-shaped area with average wind direction within $10 \mathrm{~s}$ before the moment the smell measured as axial direction and $\beta$ as deflection angle, of which the selection of deflection angle refers to the reference [16], and the deflection angle is associated with the variance of the wind direction within 10s, its specific values as shown:

$$
\beta=90^{\circ} \times S
$$

The probability distribution of Odor source at each point in the wedge-shaped area obeys the following rules. In X axis, the probability of odor source obeys normal distribution; in $\mathrm{Y}$ axis, the point is nearer to the robot, its probability to be odor source is greater, the selection of its parameters refers to concentration field formula (3). The specific implementation process as shown in Figure 2-2.

$$
c(x, y, z)=\frac{Q}{4 \pi K d} \frac{1}{d} \exp \left[-\frac{u}{2 K}(d-x)\right]
$$

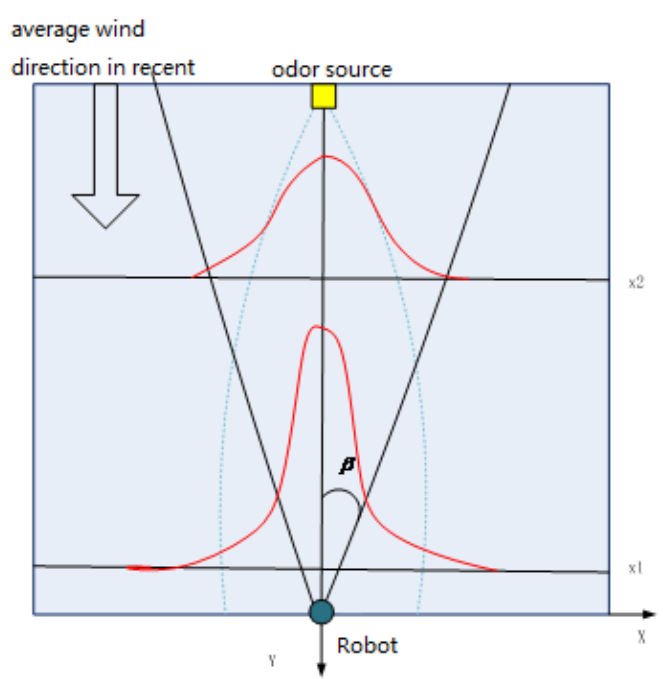

Figure 2: The inverse kinematic model of plume

The $\mathrm{X}$-axis is the average wind direction in Figure 2, the $\mathrm{Y}$-axis is direction that perpendicular to the average wind direction, it represents the width of the plume. Black thick solid line represent the possible range of odor sources which the robot measured the odor then reversed. Probability probability distribution is Trellis Plume Model reverse distribution, it represent by solid red curve in the figure, plume zone is represent by light blue dotted curve. The red rectangle is the odor source; dark blue circle represents the robot.

From the formula (1), requiring the distribution of the probability of the odor source, first obtain the turbulent diffusivity D, the literature [17], shows that the turbulent diffusivity $\mathrm{D}$ is the diffusion distance ${ }^{X}$, Reynolds $K\left(z_{0}\right)$, turbulent diffusion coefficient decision $a, b, p, q$.

$$
\begin{gathered}
D=K\left(z_{0}\right) \sqrt{\left(a x^{b}\right)^{2}+\left(10^{p} x^{q}\right)^{2}} \\
K\left(z_{0}\right)=\left(10 z_{0}\right)^{0.533 x-0.22}
\end{gathered}
$$




\section{International Journal of Science and Research (IJSR) \\ ISSN (Online): 2319-7064}

Index Copernicus Value (2013): 6.14 | Impact Factor (2015): 6.391

Where, $a=0.306-0.0302 W, b=0.940-0.044 W$, $p=-0.097-0.185 W, q=0.89$. The values of these diffusion coefficients in different directions are related with the wind speed $W$. The value of $W$ is $1,2,3,4,5,6$; its corresponding average wind speed in turn is $0-1 \mathrm{~m} / \mathrm{s}, 1-1.5$ $\mathrm{m} / \mathrm{s}, 1.5-2 \mathrm{~m} / \mathrm{s}, 2-2.5 \mathrm{~m} / \mathrm{s}, 2.5-3 \mathrm{~m} / \mathrm{s}$, and greater than $3 \mathrm{~m} / \mathrm{s}$. $z_{0}=0.014 h, h$ is the height of sensors in the robot, in the paper it's $0.8 \mathrm{~m}$.

\section{The Verification of Inverse Kinematics Model of Air Quality Mass}

In order to verify the effectiveness of the analytical model of odor sensory information a computer simulation platform has been used for the experiment. As shown in Figure (3), computer simulation platform completed by this research group early can better simulate the outdoor flow field environment, Which has shown that there is a single odor source variable airflow environment in simulation outdoor, and the moment the plume released from the odor source (composed of many black dots of varying sizes) spreads along with the flow field diffusion through time. The platform may set the location of the odor source and generates a corresponding plume according to the requirement, to meet computer simulation for robot odor localization

The platform can change wind speed and direction in real time to simulate outdoor wind field environment through time, generate plume stripes changing along with the changes of wind speed and direction, in order to show the actual winding and intermittent plume.

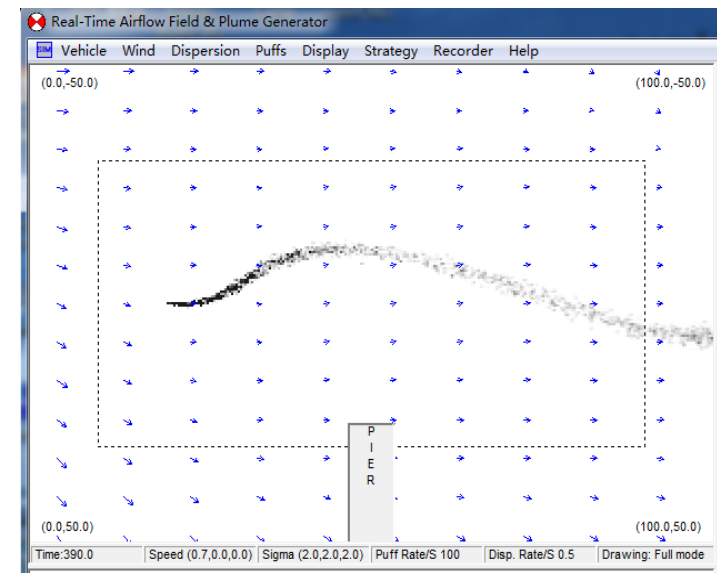

Figure 3: Computer simulation platform for odor source localization constructed by our group in early study

Figure (4) is the grayscale gained from that the robot analyse olfactory sense information in the emulational flow field environment, it verifies the results of the proposed sensor information model. In Figure (4), odor source is located in (20, $50) \mathrm{m}$, it shows that the plume strip representing by the black dots presents a winding state along with the change of the wind direction. The average wind speed of flow field is $1 \mathrm{~m} / \mathrm{s}$, the wind direction is variable through time, which is indicated by the black arrow, the direction of the arrow represents the wind direction, the length represents the size of the wind speed. The yellow circle indicates robot is stationary at point $(50,30) \mathrm{m}$.
The wind speed and direction, concentration and other information are captured in real time, and probability values that odor source appears in each grid of the upwind wedge-shaped section have been calculated.

In Figure (4), the gray grid represents probability expression of air quality mass's historical path at the current time to reach the robot. The color is darker; indicating the probability of air quality mass to pass over is greater.

The figure has shown that the grid of a triangular wedge-shaped area on the robot upwind has been marked with grayscale. The distance from the robot is closer, the grid color is darker. The grid colors in axis of average wind direction also are darker than the colors of both sides of the grid, reflecting the inverse kinematics model shown in Figure (4).

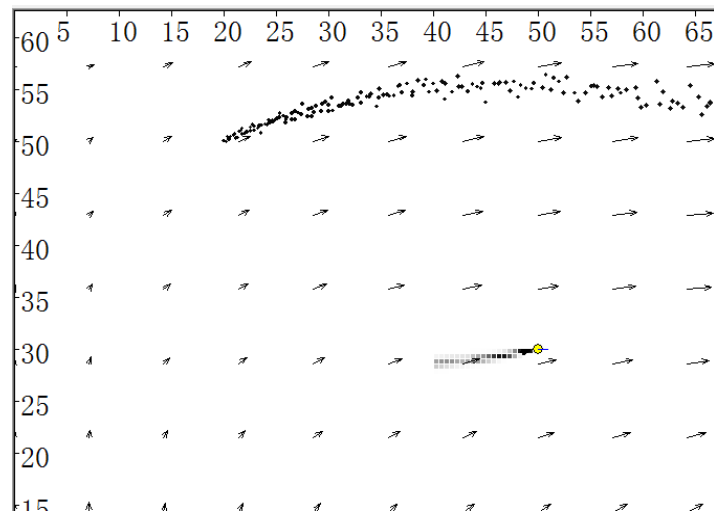

Figure 4: The simulation and verification of inverse kinematics model of air quality mass

\section{Conclusion}

This paper has introduced Lattice plume model often used in the robot odor localization studies. This paper also has introduced the simulation platform, focusing on the establishment principle of analytical model of odor sensing information, which is based on the essentially invariability of wind speed and direction in the robot's local range within 10s, and diffusion of plume within this range is mainly influenced by the average wind direction. The plume is wedge-shaped, and the analysis of sensor information is reverse parsing process of plume model. And the model has been simulated and verified on a computer simulation platform.

\section{References}

[1] Ishida H, Suetsugu K, Nakamoto T, et al. Study of autonomous mobile sensing system for localization of odor source using gas sensors and anemometric sensors [J]. Sensors and Actuators A: Physical. 1994, 45(2): 153-157.

[2] Farrell J A, Pang S, Li W. Chemical plume tracing via an autonomous underwater vehicle [J]. IEEE Journal of Oceanic Engineering. 2005, 30(2): 428-442.

[3] Holland O, Melhuish C. Some adaptive movements of animats with single symmetrical sensors[C]. Massachusetts, USA: MIT Press, 1996. 
[4] Russell R A, Bab Hadiashar A, Shepherd R L, et al. A comparison of reactive robot chemotaxis algorithms [J]. Robotics and Autonomous Systems. 2003, 45(2): 83-97.

[5] Li W, Farrell J A, Pang S, et al. Moth-inspired chemical plume tracing on an autonomous underwater vehicle[J]. IEEE Transactions on Robotics. 2006, 22(2): 292-307.

[6] Vergassola M, Villermaux E, Shraiman B I. 'Infotaxis' as a strategy for searching without gradients[J]. Nature. 2007, 445(7126): 406-409.

[7] Zarzhitsky D V, Spears D F, Thayer D R. Experimental studies of swarm robotic chemical plume tracing using computational fluid dynamics simulations[J]. International Journal of Intelligent Computing and Cybernetics. 2010, 3(4): 631-671.

[8] Murlis J, Payne T L, Birch M C, et al. Mechanisms in insect olfaction [M]. Oxford: Oxford University Press, 1986.

[9] Ferri G, Jakuba M V, Mondini A, et al. Mapping multiple gas/odor sources in an uncontrolled indoor environment using a Bayesian occupancy grid mapping based method[J]. Robotics and Autonomous Systems. 2011, 11(59): 988-1000.

[10] Kowadlo G, Russell R A. Improving the robustness of naive physics airflow mapping, using Bayesian reasoning on a multiple hypothesis tree[J]. Robotics and Autonomous Systems. 2009, 57(6-7): 723-737.

[11] Turduev M, Cabrita G, Kirtay M, et al. Experimental studies on chemical concentration map building by a multi-robot system using bio-inspired algorithms[J]. Autonomous Agents and Multi-Agent Systems. 2014, 28(1): 72-100.

[12] Monroy J G, Blanco J, Gonzalez-Jimenez J. Time-variant gas distribution mapping with obstacle information [J]. Autonomous Robots. 2015: 1-16.

[13] Meng Q H, Yang W X, Wang Y, et al. Collective Odor Source Estimation and Search in Time-Variant Airflow Environments Using Mobile Robots[J]. Sensors. 2011, 11(11): 10415-10443.

[14] Li J G, Meng Q H, Wang Y, et al. Odor source localization using a mobile robot in outdoor airflow environments with a particle filter algorithm [J]. Autonomous Robots. 2011, 30(3): 281-292.

[15] Jatmiko W, Nugraha A, Effendi R, et al. Localizing Multiple odor Sources in a dynamic environment based on Modified niche Particle Swarm Optimization with flow of wind [J]. WSEAS Transactions on Systems. 2009, 8(11): 1187-1196.

[16] Patrick P. Neumann, Victor Hernandez Bennetts. Gas source localization with a micro-drone using bio-inspired and particle filter-based algorithms. Advanced Robotics, 2013, 27: 725-738.

[17] Jie-yong Zhou, Ji-gong Li and Shi-gang Cui. A Bionic Plume Tracing Method with a Mobile Robot in Outdoor Time-varying Airflow Environment. Proceeding of the 2015 IEEE International Conference on Information and Automation Lijing, China, August 2015, 2351-2355. 